\title{
Perfiles docentes a partir de una etnografía en la escuela. Investigación acción desde el Prácticum
}

\author{
Práxedes MuÑoz SÁNCHEZ \\ Universidad Católica San Antonio de Murcia \\ pmunoz@ucam.edu \\ Cecilia Mateo SÁNChez \\ Universidad Católica San Antonio de Murcia \\ cmateo@ucam.edu \\ Ma Mercedes Álvarez García \\ Universidad Católica San Antonio de Murcia \\ malvarez@ucam.edu
}

\section{Resumen:}

Desde la antropología se presenta una etnografía realizada en escuelas unitarias, en contraste con una escuela urbana en un contexto de exclusión social en la región de Murcia. Se encontraron respuestas a la existencia de perfiles docentes que deben visibilizarse en la formación del profesorado, partiendo de las realidades educativas diversas que deben de ser analizadas en los grados de Infantil y Primaria. Mediante la etnografía reflexiva y una investigación acción participante en el Practicum, vivenciamos la necesidad de repensar la formación que se obtiene en el ámbito académico.

Palabras claves: Perfiles docentes; Practicum; etnografía reflexiva; Investigación acción participante; escuelas unitarias; Murcia.

\section{An ethnographic process at school: teacher's profiles. Participatory Action Research at Practicum}

\begin{abstract}
:
From antrohropology we emphasize this particular ethnographic process developed in multigrade schools showing the contrast with a different experience implemented at an urban school from a deprived area in Murcia. From that point the aim is to find defined teacher profiles whose contributions affect positively the teacher's training, considering the great diversity of teaching realities which should be analized in nursery and primary school education.

Through this ethnographical process of reflection as well as the Participatory Action Research at Practicum, we experienced the need of rethinking the pegagogical contents gained by the teachers at an academical training.
\end{abstract}

Key Words: Teacher's profiles; Practicum; ethnographical process of reflection; Participatory Action Research; multigrade school; Murcia.

\section{Referencia normalizada:}

Muñoz Sánchez, P., Mateo Sánchez, C. y Álvarez García, M. M. (2014): Perfiles docentes a partir de una etnografía en la escuela. Investigación acción desde el Prácticum. Historia y Comunicación Social. Vol. 19. Núm. Especial Marzo. Págs. 363-374. 
Sumario: 1.Introducción. 2. Contexto de los parámetros educativos. 3. Etnografía doblemente reflexiva en la praxis de la formación de docentes. 4. Saberes docentes en virtud de los perfiles docentes. 5. Visualizando perfiles docentes. 6 . Conclusiones.

\section{Introducción}

A continuación se presenta una etnografía realizada en escuelas unitarias, en contraste con la experiencia llevada a cabo en una escuela urbana con riesgo y caracterizada como de exclusión social en la región de Murcia ${ }^{1}$.

Desde esta etnografía en la escuela se pretende encontrar respuestas a la evidencia de perfiles docentes que permeen en la formación del profesorado, partiendo de las realidades educativas múltiples y diversas que deben de ser analizadas en la formación del profesorado en los grados de Infantil y Primaria.

Partimos de la hipótesis de que necesitamos acercarnos a la realidad cotidiana en las escuelas, para vivenciar el desarrollo día a día de los objetivos de una escuela inclusiva, y abrirnos paso desde la misma academia en los retos diarios, en conseguir los objetivos principales de solventar discriminaciones en la sociedad y en la escuela, admitir y practicar que es la felicidad de cada discente importante en la enseñanza y aprendizaje junto al éxito de la calidad en la escuela incorporando los conocimientos básicos, curriculares y extracurriculares que desarrollen cada realidad educativa.

Este proceso etnográfico va de la mano de un acompañamiento reflexivo del investigador, que hila el trabajo de campo en diferentes fases para facilitar la construcción de las ideas; cargadas éstas de consideraciones reflexivas. Como advierte Velasco \& Díaz de la Rada (1997: 23) "la originalidad metodológica consiste en la implicación del propio investigador en el trabajo, en su auto-instrumentalización". Y Hammersley y Atkinson (1994) apuestan por una etnografía reflexiva frente a paradigmas alejados de consideraciones de tipo sociocrítico:

Ni el positivismo ni el naturalismo nos proporciona una estructura adecuada para la investigación social. Ambos desconsideran su reflexividad fundamental, el hecho de que hacemos parte del mundo que estamos estudiando, y que no hay cómo escapar a la inevitabilidad de confiar en el conocimiento del sentido común y en métodos de investigación basados en el sentido común. (...) Redefinir la investigación social

1 Esta experiencia parte de una investigación etnográfica en escuelas unitarias de la Región de Murcia en España, realizado en la Universidad Católica San Antonio de Murcia, en España. Este proyecto parte de la coordinación del Practicum en los Grados de Educación Infantil y Primaria, además de un convenio de colaboración con la Dra. Lucila Galván Mora. Benemérita Escuela Normal Veracruzana “Enrique C. Rébsamen”, en México, protagonista de etnografías de educación en escuelas multigrados (Galván, Muñoz y Álvarez, 2013 y Mateo, Muñoz y Álvarez 2013). 
en términos de su reflexividad también ilumina la comprensión de la función de la etnografía (Hammersley y Atkinson 1994: 40).

Este proyecto parte de las necesidades de reflexionar en crear acciones en la formación de docentes que inviertan lo teórico con lo práctico y que creen de ésta lo teórico, a partir de las vivencias de los docentes en el campo educativo. Para esto se presenta una experiencia de saberes que se adquieren en el Practicum de la formación durante los grados, y es esta enseñanza la que permite vincular la formación con la realidad, desde un compromiso que facilite una educación social desde una voluntad propio del alumnado en el Practicum, momento para reflexionar la experimentación de lo aprendido durante el Grado de Educación:

Si hace tan sólo unos años el conocimiento de la teoría y de los contenidos de las materias que el profesor impartía era prácticamente el único requisito social y académicamente exigible al profesor, en la actualidad la realidad es mucho más compleja. Porque la teoría se entiende y valora más si se pone en relación y se construye sobre experiencias reales vividas por los alumnos (Pérez, 2010: 302)

\section{Contexto de los parámetros educativos.}

En estos tiempos convulsos de crisis, de reformas, de recortes, etc., se sigue hablando y cuestionando la tarea - misión del cuerpo de profesores, en todos los niveles educativos, desde la educación infantil hasta la universidad. Unos y otros están siempre en el ojo del huracán, precisamente por la importancia sociocultural que ejercen.

Los docentes sufren todo tipo de exigencias y de ataques por parte de la sociedad en la que viven, prueba de ello son, desde el fracaso escolar hasta la urgencia de la innovación educativa que concurre con las nuevas tecnologías, pasando por las cuestiones de salud, de género, etc. Estos temas también están reflejados en las temáticas finales de los trabajos del alumnado de los Grados de Educación Infantil y Primaria, dando muestra de las vías que le son marcadas, precisamente en el Practicum, por las experiencias vividas en el mismo, y que son así mismo entendemos un reflejo de los perfiles docentes que se van marcando, obviando otras formas presentes e innovadoras. Ahora bien, consideramos que no hay nada más innovador en la educación en este mundo líquido, parafraseando a Bauman (2013) que ser uno mismo, en la mayor plenitud como ser humano, como persona, como profesional y evidentemente como docente vocacional, pues de ese desarrollo podremos asegurar que el perfil de docente de siglo XXI estará en línea de seguir dando respuesta a la única demanda social que consideramos importante: humanizar.

Co-ayudar en la humanización de la persona en desarrollo, es tan claro y tan antiguo como ya ponía de manifiesto Platón en Menón. Pues no habrá educador que no haya descubierto en su trayectoria personal y profesional, que no hay enseñanza sin aprendizaje, y que esté pasa irremediablemente por el conocimiento de uno mismo, 
poniendo en ese proceso de humanización, todas sus posibilidades, competencias y facultades que como persona tiene en sí misma.

Los futuros docentes en su trayectoria de formación inicial, han de vislumbrar que su formación va en paralelo a la del grupo de discentes que tienen ante sí. Pues no tienen nada que enseñar, sino de acompañar el proceso, de guiar en el camino de la libertad como personas, practicando tal vez el tercer nivel de educación puesto de manifiesto por Bateson (1998), y que posibilita desmembrar y volver a organizar o no el sistema marco predominante de una sociedad, en aras a la libertad a la que estamos llamados como seres humanos (Alain 2002).

Los estudiantes de los Grados de Educación se encuentran en el transcurso de sus prácticas con la tarea - misión apasionante de enseñar, siendo también consciente de la visión utilitarista que la sociedad, perdiendo con ella la esencia, de lo que Píndaro ya nos dejaba en su lema "llega a ser lo que eres", en el que sin duda tienen mucho que hacer con sus discentes en este momento presente, tan necesitado de los mejores docentes.

Apostamos por recuperar las prácticas educativas como experiencias: "según es vivida por sus protagonistas y atendiendo a lo que les supone, a lo que les significa" (Contreras y Pérez, 2010, Pág. 22). Considero importante lo que recuperan los autores José Contreras y Nuria Pérez (2010) en el caso de la experiencia a la práctica y su vuelta, es decir, devolver en un ejercicio de actividades educativas, o de buenas prácticas, para responder a una educación de calidad y equitativa. Esto debe de ir acompañado de una parte de vocación, donde lo personal es vital y necesario de reconocer (Montoya, 2002). Así, otra de las apuestas es por una pedagogía desde prácticas feministas, en primer lugar desde un cuidado en el lenguaje no sexista, no discriminatorio, que protagoniza las emociones, lo vivido e incluso lo espiritual en la noción de una educación meritoria que nombra lo vivido, donde es el día a día con la comunidad educativa, en la educación formal y no formal, lo privado de cada individuo se hace público, preocupada por una educación social.

El cambio en el que nos centramos es desde la conciencia que alivia la oportunidad de la singularidad y la atención de cada uno y una, docente, discente y realidad social. Por esto, este artículo protagoniza la necesidad de repensar la educación, la realidad existente y qué protagonizar de los posibles perfiles profesionales.

\section{Etnografía doblemente reflexiva en la praxis de la formación de docentes}

El planteamiento de la investigación ha sido realizar una etnografía reflexiva incorporando como sujetos de investigación a la comunidad educativa y las propias investigadoras, coordinadoras y partícipes del Practicum en el Grado de Educación Infantil y Primaria. 
El objetivo era repensar la educación a partir de los saberes docentes (Galván, 2012). A lo que añadimos los saberes de toda la comunidad educativa, haciendo partícipe a las familias, al alumnado e incluso exalumnado, desde un enfoque como proceso etnográfico, con la propuesta de investigación acción, ya que se provoca a partir de crear y despertar lo teórico desde lo cotidiano, invitando a los actuales docentes a que compartan su espacio de reflexión, a la vez que creándolo en seminarios interinstitucionales del ámbito escolar y social, para pensar y repensar nuestro sistema educativo en la práctica y nuestra formación de futuros docentes en los Grados de Educación Infantil y Primaria, junto a los Practicos.

La etnografía es un proceso metodológico que está inmersa en los objetivos de la investigación, convirtiéndose éste en un objetivo del encuentro de lo experiencial con el conocimiento dejando entrever una etnografía en primera persona (Galván, Muñoz y Álvarez, 2013).

Es evidente que en la etnografía se cuenta con el proceso personal de las autoras, porque lo consideramos parte de las resultados obtenidos, principalmente cada una ha permeado sus disciplinas así como perspectivas personales aprendidas incluso en este proceso como en la formación de profesorado y las experiencias encontradas en el campo, es decir en las escuelas reales de este primer encuentro.

Este trabajo se inicia considerando que la etnografía aporta un conocimiento de realidades y apostando por la escuela unitaria para enlazar saberes de la atención a la diversidad desde multiniveles en cada una de las aulas, en este caso una de primaria y otra de infantil, seleccionamos diferentes escuelas. Buscamos una escuela unitaria alejada de los centros urbanos donde un alumno del Grado de Educación Primaria podía desarrollar su Practicum atraído por estar cerca de la naturaleza, en este caso en Sierra Espuña. Este alumno ya venía de hacer el Practicum I en un colegio público urbano con características de una comunidad educativa especialmente estigmatizada de exclusión social en un barrio suburbial de Cartagena.

La selección escogida del propio alumno del Practicum ${ }^{2}$, fue tan compleja y diversa como la realidad educativa es. Esto nos dio confianza en su compromiso con la educación, necesitaba algo que la propia formación como maestro de Primaria no había conseguido o pensaba que no era suficiente, a la vez que estaba sediento por comprender qué estrategias educativas son las que se deben hacer para construir la inclusión socioeducativa, y a la vez, con un entorno comunitario que mantiene valores de su cuidado: "algo más profundo del significante investigar que no puede encerrarse en los muros universitarios" (López y Caramés, 2010: 113).

El objetivo de la etnografía también era ser vehicular haciendo etnografía en primera persona, y nace con voluntad de acción en una antropología comprometida

2 Agradecemos la abertura, flexibilidad y ganas de aprender del alumno en el Practicum, Luis Suárez, alumno graduado en 2013 como maestro de primaria en la UCAM, quien nos ha acompañado en este proceso, tanto de él en el Practicum como en la investigación en la escuelas unitarias. Damos fe de su compromiso por la educación, que quiere dedicar, mayormente, a evitar la exclusión social. 
(Hale 2008 y Muñoz 2012) que quiere visibilizar qué se está haciendo para mejorar la educación y apostar por construir comunidades educativas que no favorezcan barreras con la sociedad más cercana, y a la vez, fomentar encuentros de diálogo en esta reflexión en conjunto, propiciando la doble reflexividad requerida desde la educación política, y así obtener desde la praxis y acercar esta apuesta a los espacios académicos.

La etnografía consistió en realizar entrevistas en profundidad, e historias de vida de directores de los centros y otros docentes, talleres entre las maestras, talleres con alumnado de primaria y talleres con alumnado de infantil, entrevistas a exalumnos y por último talleres con madres del alumnado. La investigación más completa fue en la CEIP. El Berro, en Sierra Espuña, perteneciente al municipio de Alhama de Murcia, en los CEIP. Miguel Hernández y CEIP. La Matanza, se realizó con menos detalle por no tener alumnado ejerciendo el Practicum. Por último, añadimos el Practicum en el CEIP de Lo Campano, donde el alumno pudo realizar observación sistemática y participante, y redactar su cuaderno de campo.

El alumno del Practicum convivió profundamente con las maestras del centro. Al tener que compartir el mismo transporte que la directora, le permitió una relación más cercana, así como quedándose en las tardes y en horas de tutoría y administración del centro. Esto le permitió conocer mejor los objetivos y competencias de la educación, involucrándose en la enseñanza y en su preparación, como en la práctica de la vida educativa. Este alumno participó en la parte etnográfica como de tutelado, ambas respuestas de la universidad favorecieron que entendiera y le diera sentido a la necesidad de invertir este tiempo en abrir espacios a la investigación, algo interesante de iniciar desde el primer año de formación en el Grado.

Se crea así una "simbiosis entre la etnografía reflexiva y la investigación-acción" (Muñoz, 2009, Pág. 200). La investigación será un análisis que desea despertar la doble hermenéutica dentro del proceso de investigación entre el etnógrafo y los “"expertos' de su propio mundo de vida" (Dietz 2003: 142), o como nos dice Martha Caramés (2010: 200): “¿cómo es posible ser educador, maestra o profesor en la universidad sin espacio para aprender?".

\section{Saberes docentes en virtud de los perfiles docentes}

Los saberes docentes son en primer lugar las experiencias y las vidas de muchos docentes en situaciones singulares y cada una especial en la complejidad educativa. La decisión de emprender un proyecto sobre la construcción de los saberes docentes, deriva de un interés compartido por poner de manifiesto el conocimiento profesional que los docentes generan en una situación de enseñanza multigrado (escuelas unitarias); en escuelas ubicadas en zonas rurales de difícil acceso y en condiciones no siempre adecuadas para su labor, en este caso en contextos de exclusión social. A pesar de que los sistemas educativos de nuestros países cuentan con buen número 
de estas escuelas, éstas han sido invisibles, tanto para las políticas educativas que pasan por alto sus condiciones y especificidad, como para la investigación que recién empieza a ubicarlas entre los temas de interés.

El estudio de los saberes docentes, desde una perspectiva etnográfica, parte de una noción que destaca el vínculo entre el saber y la experiencia cotidiana del profesorado, al que añadimos procesos reflexivos que acompañan la enseñanza y los contextos institucionales que enmarcan el trabajo docente. Integrados a la práctica cotidiana en el aula, los saberes forman parte de la institución escolar y, como conocimiento generado frente a problemas de trabajo propios de la profesión, constituyen un patrimonio histórico y social que es necesario valorar (Rockwell y Mercado, 2003).

Los saberes docentes o saberes profesionales son tan diversos como cada singularidad de cualquier docente (con mayúsculas), aquí señalamos que algo esencial es que se requiere profesionales con oficios, con un posible éxito que responde a un determinado perfil de personalidad y a buenas prácticas iniciales (Pérez, 2010).

Muchas de los saberes docentes cambian según el contexto, el momento histórico, la política educativa y la base del sistema y la estructura educativa, por tanto, los perfiles docentes van a la par de las acciones posibles en la educación.

La etnografía realizada ha estado vinculada a encontrar una relación libre y lo más justa posible de la educación formativa de maestros y la realidad que se van encontrar en el aula, con un ansia por hallar perfiles docentes singulares y a la vez complejos, en las múltiples realidades socioeducativas. Es fruto de una sed de evidenciar si los contenidos y competencias adquiridas serán válidos para ejercer de docente.

Este proceso etnográfico se convirtió en una investigación acción participativa de toda la comunidad educativa, destacamos la escuela unitaria del Berro, porque atraíamos a vecinos y familia a esta reflexión de la educación, recuperando la historia en la escuela unitaria y los procesos de formación y experiencia. En la escuela del municipio de Cartagena, fue un proceso del alumnado del Practicum junto a un trabajo realizado por alumnos de la asignatura de Atención a la Diversidad e Interculturalidad, que en su segundo curso del Grado de Primaria, se adentraron a la realidad educativa, cuya experiencia fue un despertar de saberes y perfiles docentes, así como delata esta afirmación: "se pueden hacer muchas cosas con alumnos tan complicados", "podría trabajar en un contexto de exclusión".

\section{Visualizando perfiles docentes.}

En este apartado presentamos las inspiraciones encontradas en el proceso etnográfico y en la investigación acción participante, que ha ido construyendo este encuentro de saberes. Desde la visión como sensación por doquier de que la universidad, lo

3 Alumno de $2^{\circ}$ del grado de Primaria, 2011-2012. UCAM. 
académico, está alejado de transferir conocimientos empíricos, y no es porque no se hace suficientemente, sino porque los contenidos son exigidos para un espacio de tiempo corto, y es casi imposible dejar un espacio de reflexión con profesorado de los grados que se imparten. Esto puede solventarse distribuyendo el Practicum en todos los años de formación y no solo al final de los cursos, sumando la participación en visibilizar las prácticas educativas, por ejemplo en jornadas abiertas de centros con experiencia en una atención a la diversidad y ejemplos de educación inclusiva, asimismo como aquellos centros con más dificultades y alejados de esta educación inclusiva.

Lo analizado en cada uno de las realidades educativas, junto a visibilizar las intenciones realizadas y algunas más conocidas desde las experiencias vividas en formación, reflexionamos sobre las acciones que pueden adaptarse a propuestas academicistas en la formación del profesorado.

En primer lugar realizamos un estudio sobre las dificultades encontradas que favorecen la desigualdad y exclusión social, y otras que las hace más favorables (Suárez, Muñoz y Álvarez, 2013). Nos abre paso a perfiles tan diversos como realidades existen. Exponemos las estrategias identificadas en esta etnografía (poniendo aquí las más importantes y significativas):

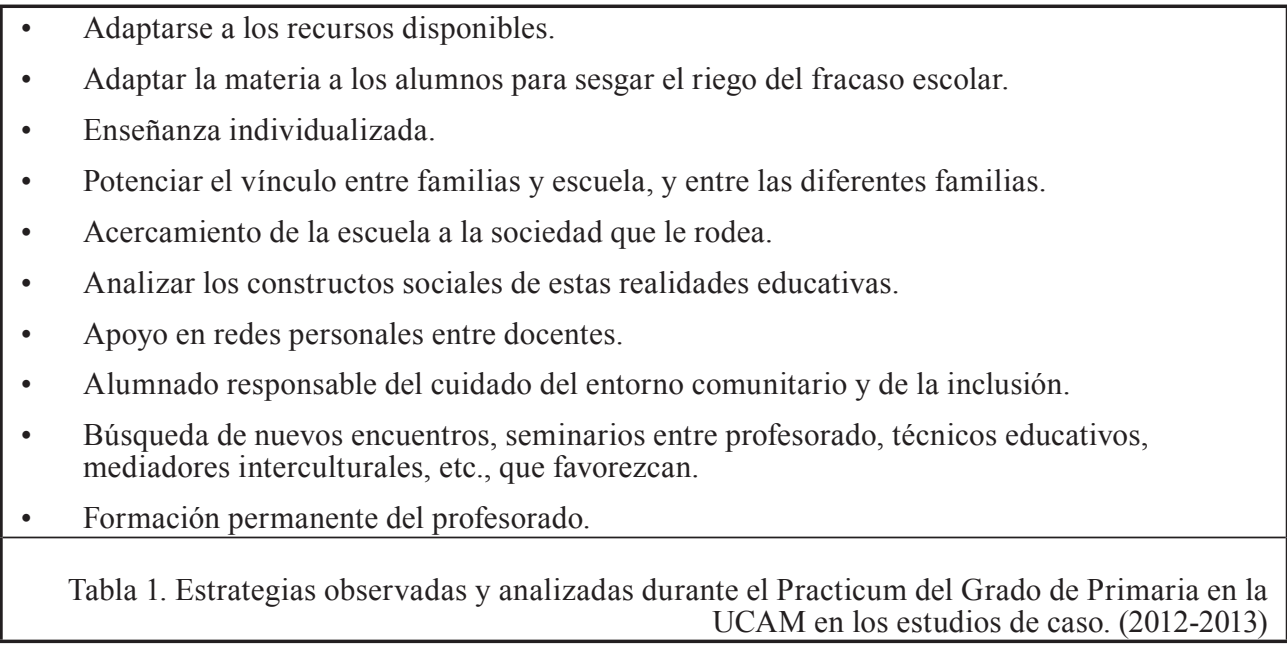

Toda esta responsabilidad se revierte en una educación inclusiva., pero en este espacio recuperamos los saberes docentes desde la reflexividad de la comunidad educativa junto a las autoras de la experiencia investigativa. Es decir, cómo realizan su docencia, cómo se atiende a la diversidad, qué nuevas metodologías están aportando y si es necesario que se aplique a la formación del profesorado así como a futuros maestros de infantil y primaria. 
En la etnografía constatamos que existe un perfil docente de la escuela unitaria así como de escuelas en contextos de exclusión social, y ha sido la incursión en las aulas, en los colegios y en los pueblos y barrios cuando se a partir de una etnografía reflexiva que se han identificado procesos incluso personales de cada docente, que está vinculado a los proyectos educativos de centro, como a la realidad socioeducativa de la escuela.

Aun así, desde una investigación acción participante, se organizaron seminarios científicos en la universidad sobre estos saberes docentes, para ser escuchados por el alumnado así como por el claustro de Educación

Consideramos como temas principales a tratar los siguientes:

- Contextualización del centro: historia, identidad, comunidad educativa, etc.

- Estrategias y recursos: procesos de enseñanza, tareas, actividades, formación del profesorado, etc.

- Perfil del docente: identidad, actitud, competencias, etc.

- Líneas de acción y futuro.

Para esto, nos reforzamos en el el "diálogo de saberes" que construye el autor Gunther Dietz (2012) en México, así como Lucila Galván (2012), cuyo enfoque está en repensar la educación. La autora Ruth Mercado (2002) avisa del carácter histórico y dialógico de saberes, incluyendo la dimensión social. A lo que sumamos en el saber cotidiano, que incluso ha sido un descubrimiento para el alumno del Practicum, debido a la idealización que tenía de cómo son las escuelas rurales y en ámbitos de montaña. Y ha sido desde este encuentro que ha puesto en acción su observación sistemática y participante, para revelar el saber cotidiano, experiencial, determinante en cada contexto y con cada docente y discente, revelando la creatividad pedagógica, el pensamiento reflexivo y la capacidad innovadora que los docentes sitúan en determinados contextos educativos, porque éstos actúan en zonas indeterminadas de la práctica (Schón, 1998).

\section{Conclusiones}

(...) El alumno mentor a veces es espontáneo, no siempre preparado, aprovecha la situación como comunidad de aprendizaje. (...) El perfil del rol de la unitaria es que es difícil cuando en un curso tienen diferentes etapas. Yo trabajaría el interés de la escuela y no se trabaja. Aprendizaje a través del interés del niño se podría hacer, pero "hay miedo a escalones" (del conocimiento o currricular) La escuela hace lo que dice el inspector, el inspector del gobierno y más que pensar en qué aprender del curriculum: "pero la preocupación es el inspector". (Entrevista Alumno Practicum L. Suárez marzo 2013)

Tras la visita y la inserción en varios colegios, tomamos conciencia de la realidad educativa que atañe a estos centros, la cual se presenta a través de una etnogra- 
fía. Esta realidad, no deja impasible ni al alumnado, ni al docente, cuanto menos al investigador. El día a día, de esta comunidad educativa se exhibe a simple vista con un presente que aparentemente, puede resultar muy complejo para algunos futuros docentes, y para otros puede resultar un dulce, un manjar, solo se aleja de la cotidianeidad en que los espacios educativos están alejados de los medios de comunicación, de los intereses de futuros docentes, porque se escapan a realidades, se quedan en minorías muy invisibilizadas.

Estos pequeños centros ofrecen una visión muy enriquecedora de las competencias necesarias para el desempeño de la labor docente, así como un amplio conocimiento de la interculturalidad que se respira una vez sobrepasado el umbral de la puerta de acceso, muchos repletos de la vocación docente que realza María Zambrano (2007), así como en el entusiasmo simbólico de maestros y maestras audaces que siguen adelante a pesar de todo (Nieto, 2006).

Nuestra apuesta es por evitar en la formación de profesorado contenidos generalistas y concretar en la realidad existente, enfocándose en adquirir habilidades y competencias necesarias para cada realidad educativa. Esta puede ser enfocada desde menciones a la diversidad de contextos educativos, y dejar el homogéneo porque no es real, partir de la complejidad educativa y construir espacios de estrategias educativas, y creer en posibilitar una investigación acción participante desde la universidad. Y por último, fomentar un voluntariado que desde primer grado, se formalice un compromiso para estar activo en mejorar la educación desde cada contexto, aprendiendo desde saberes y añadiendo creatividad y las motivaciones de nóveles maestros.

\section{Bibliografía}

CARAMÉS, M. (2010). Investigar en educación: Un espacio posible y sobre todo imposible. En CONTRERAS, J. y PÉREZ DE LARA, N. (Comps.) (2010). Investigar la experiencia educativa. Madrid: Morata. p. 199-210.

CONTRERAS, J. y PÉREZ DE LARA, N. (comps.) (2010). Investigar la experiencia educativa. Madrid: Morata.

DIETZ, G. (2003). Multiculturalismo, Interculturalidad y Educación: Una aproximación antropológica. Granada: CIESAS. Universidad de Granada.

DIETZ, G. (2012). Reflexividad y diálogo en etnografía colaborativa: el acompañamiento etnográfico de una institución educativa intercultural mexicana. Revista de Antropología Social no 21,Madrid: AIBR. p. 63-91.

GALVÁN MORA, L. (2012). Enigmas y Dilemas de la Práctica Docente. Barcelona: Octaedro.

GALVÁN MORA, L.; MUÑOZ SÁNCHEZ, P. y ÁLVAREZ GARCÍA, M ${ }^{\mathrm{a}}$ M. (2013). Los saberes docentes en la escuela multigrado. Un estudio etnográfico en México y España. Ponencia en el III Congreso Internacional de Etnografía y Educación. Madrid. (En proceso de publicación). 
HALE, Ch. (2008). La antropología comprometida en transición. En BASTOS S. (comp.) Multiculturalismo y futuro en Guatemala. Guatemala: FLACSO/ OXFAM. p. 217-245.

LÓPEZ CARRETERO, A. y CARAMÉS I BOADA, M. (2010). La vida es una. Investigar, una práctica política. En ARNAUS, Remei y PIUSSI, Anna María (Coords.). La universidad fértil, mujeres y hombres, una apuesta política. Barcelona: Octaedro.

LÓPEZ, M. (2011), Modelos organizativos en la escuela rural de la Región de Murcia. Primer Congreso Estatal La educación en el medio rural.

MERCADO, R. (2002). Los saberes docentes como construcción social. México: Fondo de Cultura Económica.

MUÑOZ SÁNCHEZ, P. (2012). Dilemas de una antropología comprometida: entre autores y experiencias etnográficas. En PEÑA, B. (coord.) Desarrollo Humano. Madrid: Visión Libros. p. 247-281.

MUÑOZ, SÁNCHEZ, P. (2009): "De la violencia surge una cultura política de resistencia, las CPRs del Ixcán, que deriva en una participación estratégica desde las municipalidades", Vol. 5, no 8, p.196-236.

NIETO, S. (2006). Razones del profesorado para seguir con entusiasmo. Barcelona: Octaedro.

PÉREZ SÁNZ, A. (2010). El nuevo profesor de secundaria. En González I. (coord.) El nuevo profesor de secundaria. Barcelona: Grao. p. 301-309.

ROCKWELL, E. y MERCADO R. (2003). "La práctica docente y la formación de maestros", en La escuela, lugar del trabajo docente. México: DIE-CINVESTAV-IPN.

SCHÓN, D. (1998). El profesional reflexivo. Cómo piensan los profesionales cuando actúan. Barcelona: Paidós.

ZAMBRANO ALARCÓN, M. (2007) Filosofia y educación. Manuscritos. Ángel Casado y Juana Sánchez- Gey (Eds.). Málaga: Ágora.

\section{Las autoras}

Práxedes Muñoz Sánchez: Licenciada en Filosofía y letras por la Universidad de Murcia, España. Doctora en Antropología por la Universidad de Murcia (Área de Psicología Social) con el título: Las Comunidades de Población en Resistencia, CPR del Ixcán, Guatemala, el surgimiento de una nueva identidad comunitaria". Actualmente docente e investigadora en la Universidad Católica de San Antonio, Murcia, en los Grados de Educación Infantil, Educación Primaria, Antropología Social y Cultural y en el Máster de Desarrollo Social. Estancias investigadoras en Guatemala y México: Beca MAEC-AECID 2009-2011, en ECOSUR Unidad Villahermosa, México; Investigadora huésped en CIESAS Sureste, México 2009 y Beca MUTIS en Guatemala Unviersidad Landíva, 2000-2001. 
Cecilia Mateo Sánchez. Licenciada en Historia del Arte, Universidad de Murcia. Máster en Museología Contemporánea, Universidad de Alicante. Máster en Educación y nuevas tecnologías con publicación de tesina por la universidad a distancia de Madrid. Actualmente doctoranda en arte y comunicación en la UCAM. Profesora e investigadora en los Grados de Educación Infantil Primaria y Máster del profesorado y está en la dirección del Practicum en la UCAM.

$\mathrm{M}^{\mathrm{a}}$ Mercedes Álvarez García. Diploma de Estudios Avanzados- Facultad de Educación. Universidad de Murcia. Junio de 2007. Máster en Ciencias de las religionesFacultad de Filosofía- Universidad de Granada. Junio 2007. Ciencias de la Educación. Especialidad Organización y Dirección de Centros Educativos- 2002- UNED. Licenciada en Música Especialidad piano- 1981- Conservatorio de Murcia. Diplomada en Profesorado de E.G.B. Especialidad de Ciencias Sociales 1984- Universidad de Murcia. Coordinadora de los Practica de los Grados de Educación Infantil y Primaria, así como del Máster Universitario de Formación del Profesorado UCAM desde el curso 2010-2014. Profesora del Máster Universitario de Formación del Profesorado y de los Grados de Educación Infantil y Primaria de la Universidad Católica de Murcia, en la asignatura de Contextos educativos y Organización y gestión escolar. 